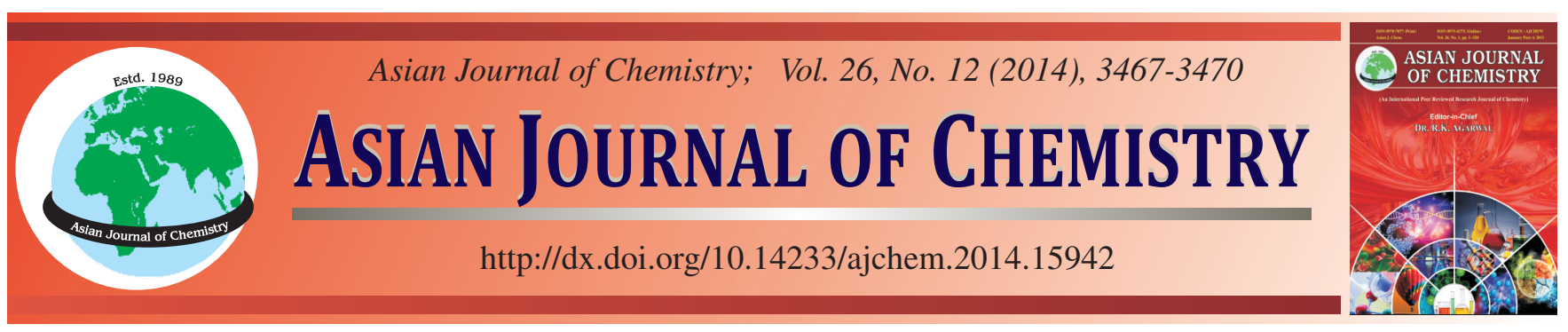

\title{
Blue Luminescent ZnO Nanoclusters Stabilized by Esterifiable Polyamidoamine Dendrimers and their UV-Shielding Applications
}

\author{
FAN ZhANG ${ }^{*}$, Bo WANG and YongQIANG SHeN
}

College of Chemistry and Chemical Engineering, Jishou University, Jishou 416000, P.R. China

*Corresponding author: Tel/Fax: +86 743 8563911; E-mail: chemfzhang@163.com; zhangfan8346@sina.com

\begin{abstract}
Zinc oxide quantum dots (QDs) were added to the water solutions of esterifiable polyamidoamine (PAMAMs) and the ZnO nanoclusters were prepared. The $\mathrm{ZnO}$ nanoclusters are stable in water and show bright blue fluorescence under UV lamp. TEM images showed that the $\mathrm{ZnO}$ nanoclusters consisted of numerous $\mathrm{ZnO}$ QDs. FTIR spectra of $\mathrm{ZnO}$ nanoclusters demonstrated the successful modification of $\mathrm{ZnO}$ QDs by the esterifiable polyamidoamine dendrimers. The UV-visible absorption spectra indicated that the ZnO nanoclusters exhibited wider and red-shifted comparing with ZnO QDs. The fluorescent spectra showed that the emission peaks at $404 \mathrm{~nm}$ of the ZnO QDs became weaker and a new emission peaks appeared at $330 \mathrm{~nm}$ after modifying the ZnO QDs with polyamidoamine dendrimers. The UVvisible transmittance spectra showed that the poly(vinyl alcohol)/ZnO nanoclusters film absorbed nearly $99 \%$ of UV light at wavelengths between 200 and $240 \mathrm{~nm}$ while $75 \%$ between 240 and $300 \mathrm{~nm}$.
\end{abstract}

Keywords: ZnO quantum dots, Nanoclusters, Polyamidoamine dendrimers, UV-shielding, Poly(vinyl alcohol).

\section{INTRODUCTION}

As one of a group of II-VI semiconductors, zinc oxide has attracted much attention for its potential applications in blue/ UV optoelectronics, transparent electronics, pintronic devices and sensor applications. Zinc oxide has been commonly used in its polycrystalline form for over a hundred years in a wide range of applications e.g., facial powders, ointments, sunscreens, catalysts, lubricant additives, paint pigmentation, piezoelectric transducers, varistors and transparent conducting electrodes ${ }^{1}$. However, the nanoscale crystals tend to aggregate or to undergo Ostwald ripening because of their high surface energy. Therefore, appropriate surface modification is needed to stabilize the $\mathrm{ZnO}$ nanocrystals ${ }^{2-10}$. Hagura et al. ${ }^{11}$ had reported that highly luminescent silica-coated $\mathrm{ZnO}$ nanoparticles dispersed in an aqueous medium were synthesized using the sol-gel process. Liu and co-workers ${ }^{12}$ described a new approach for preparation of $\mathrm{ZnO}$ nanocrystals stabilized by ionic liquid components. The ionic liquid-ZnO nanocrystal composite was formed directly from an ionic liquid salt containing $\mathrm{Zn}$ (II) cations. Xia and co-workers ${ }^{13}$ recently obtained polymer-stabilized $\mathrm{ZnO}$ nanoparticles with remarkable blue fluorescence, however, the origin of the emission was not very clear. An organic dispersion of 9-15 $\mathrm{nm}$ size dysprosium oxide incorporated zinc oxide nanocomposites exhibiting luminescence in the visible region had been synthesized by a wet chemical precipitation technique at room temperature ${ }^{14}$, and the work showed that stability and functionalization of the same nano-composites in organic medium was achieved by using 1:1 volume ratios of (tetraethoxysilane $\left[\left(\mathrm{C}_{2} \mathrm{H}_{5} \mathrm{O}\right)_{4} \mathrm{Si}\right]$, (3-aminopropyl) trimethoxysilane) as the capping agent.

Polyamidoamine dendrimers (PAMAMs) with large amounts of terminal functional groups have been paid more and more attention due to their unique features such as excellent biocompatibility, good solubility and high reactivity ${ }^{15-21}$. In this paper, esterifiable PAMAMs was chosen as modifiers, because esterifiable PAMAMs contained lots of tertiary amine, which could act as organic ligands and stabilize the $\mathrm{ZnO}$ quantum dots (QDs). Our goal is to obtain a kind of water soluble $\mathrm{ZnO}$ clusters which could be applied at the domain such as UV-shielding, medicine, biomedical material. The reports about water soluble $\mathrm{ZnO}$ clusters were little found. Here, we describe the fabrication of $\mathrm{ZnO}$-PVA nanocomposite thin films by casting homogeneous solution of $\mathrm{ZnO}$ nanoclusters and poly(vinyl alcohol) (PVA). The $\mathrm{ZnO}$ clusters were characterized by FTIR spectra, TEM, UV/visible absorbance spectra and photoluminescence (PL) emission and excitation spectra. The luminescent features of $\mathrm{ZnO}$ clusters/PVA film were also investigated.

\section{EXPERIMENTAL}

$\mathrm{Zn}\left(\mathrm{CH}_{3} \mathrm{COO}\right)_{2} \cdot 2 \mathrm{H}_{2} \mathrm{O}, \mathrm{KOH}$ and $\mathrm{CH}_{3} \mathrm{OH}$ were obtained from Tianjin Kemiou Chemical Reagent Co Ltd., in Tianjin, China. All chemicals were analytical reagent and used without further purification. 
Preparation of ZnO QDs: The preparation of ZnOQDs was based on literature ${ }^{12,22}$. Typically, $0.98 \mathrm{~g} \mathrm{Zn}\left(\mathrm{CH}_{3} \mathrm{COO}\right)_{2} \cdot 2 \mathrm{H}_{2} \mathrm{O}$ and $50 \mu \mathrm{L}$ of deionized water were added into a flask containing $40 \mathrm{~mL}$ of methanol. The solution was heated to $64{ }^{\circ} \mathrm{C}$ with vigorous magnetic stirring. $0.496 \mathrm{~g} \mathrm{KOH}$ was dissolved into $20 \mathrm{~mL}$ of methanol as the stock solution that was then added into the flask within 10-20 min. At a constant temperature of $64{ }^{\circ} \mathrm{C}$, it took $3 \mathrm{~h}$ to obtain $\mathrm{ZnO}$ QDs. When the reaction was finished, the upper fraction of the reaction solution was discarded after $120 \mathrm{~min} .50 \mathrm{~mL}$ of methanol was added to the solution and stirred for $2 \mathrm{~min}$. Again, the upper fraction of the solution was discarded after $120 \mathrm{~min}$. The methanol washing process was repeated and the upper fraction of the solution was taken away after overnight staying. The isolated $\mathrm{ZnO}$ QDs were dissolved in chloroform.

Preparation of esterifiable polyamidoamine dendrimers: Generation 2.5, 3.5, 4.5 (G2.5, G3.5, G4.5) PAMAMs were prepared according to the procedures described in thce literature $^{23,24}$, then the water solutions of PAMAMs of different half generation were prepared.

Preparation of the $\mathrm{ZnO}$ nanoclusters: A desired amount of $\mathrm{ZnO}$ QDs was added to the water solutions of PAMAMs of different half generation respectively and the $\mathrm{ZnO}$ QDs were fully dissolved in the solution very slowly about two months later, and then the $\mathrm{ZnO}$ nanoclusters were prepared. The molar ratios of ZnO QDs and 2.5G PAMAMs, 3.5G PAMAMs, 4.5G PAMAMs are 4:1, 8:1 and 16:1, respectively. The typical preparation of the $\mathrm{ZnO}$ clusters is depicted in Fig. 1.

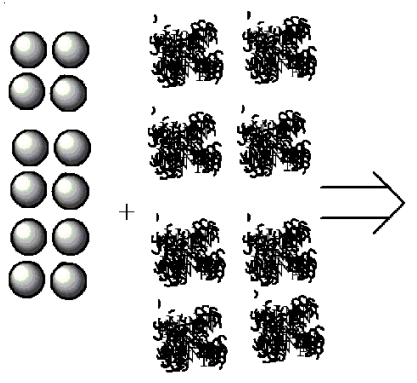

ZnO QDs 3.5GPAMAMs

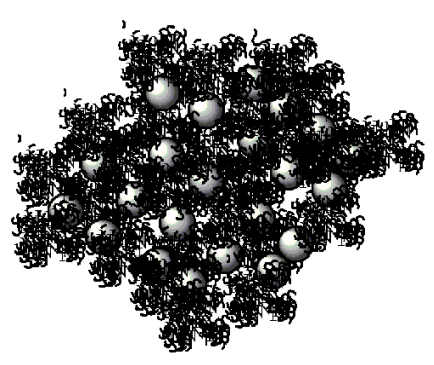

ZnO nanoclusters
Fig. 1. Schematic illustration of the preparation of the $\mathrm{ZnO}$ nanoclusters

Preparation of PVA/ ZnO nanoclusters film: The fabrication process of the PVA/ZnO nanoclusters film was depicted as follows: a desired amount of water solution of $\mathrm{ZnO}$ nanoclusters $(0.04 \mathrm{~g} \mathrm{ZnO})$ was mixed with a water solution of PVA (2 $\mathrm{g}$ PVA, $50 \mathrm{~mL}$ of deionized water), Then the intermixed solution of $\mathrm{ZnO}$ nanoclusters and PVA was casted into a petridish, followed by solvent evaporation at $30{ }^{\circ} \mathrm{C}$ under vacuum to obtain PVA/ZnO nanoclusters composite film $(200 \mu \mathrm{m}$ in thickness). The pure PVA film and PVA/ ZnO QDs composite film were obtained under the same condition as PVA/ZnO nanoclusters film, the concentrations of $\mathrm{ZnO}$ in $\mathrm{PVA} / \mathrm{ZnO}$ nanoclusters film and PVA/ZnO QDs composite film were 2 wt. $\%$.

Characterization: FTIR spectra were recorded on a IRAffinisy-1 spectrometer (Shimadzu) by using potassium bromide discs prepared from powdered samples mixed with dry $\mathrm{KBr}$. The microstructures of $\mathrm{ZnO}$ QDs and $\mathrm{ZnO}$ nanoclusters were observed by transmission electron microscopy (TEM) (HR-TEM, JEOL Model 3010F). Drops of the diluted suspension were placed onto $\mathrm{Cu}$ TEM grids. As the $\mathrm{CH}_{3} \mathrm{Cl}$ and water solvents were evaporated, $\mathrm{ZnO}$ nanoclusters and $\mathrm{ZnO}$ QDs were left on the $\mathrm{Cu}$ support.

UV/Visible absorbance spectra of $\mathrm{ZnO}$ QDs and $\mathrm{ZnO}$ nanoclusters were recorded with a Perkin Elmer Lambda-6 UVvisible spectrophotometer. Photoluminescence (PL) emission and excitation spectra of $\mathrm{ZnO}$ QDs and $\mathrm{ZnO}$ nanoclusters were recorded at room temperature by using Shimadzu-RF-5000 fluorescence spectrophotometer.

\section{RESULTS AND DISCUSSION}

Fig. 2 shows the digital photographs of a chloroform solution of $\mathrm{ZnO}$ QDs and a typical water solution of $\mathrm{ZnO}$ nanoclusters. It was found that the chloroform solution of $\mathrm{ZnO}$ QDs displayed in colorless under daylight and in yellow fluorescence under UV lamp $(\lambda=365 \mathrm{~nm})$. However, the $\mathrm{ZnO}$ nanoclusters, which modified by PAMAMs of different half generation emitted bright blue fluorescence under the excitation of UV light $(\lambda=$ $365 \mathrm{~nm}$ ). For preparing $\mathrm{ZnO}$ nanoclusters, the $\mathrm{ZnO}$ QDs were dissolved in the water solutions of PAMAMs of different half generation very slowly. The resulted water solution of $\mathrm{ZnO}$ nanoclusters could be stable even under sunlight over a year.

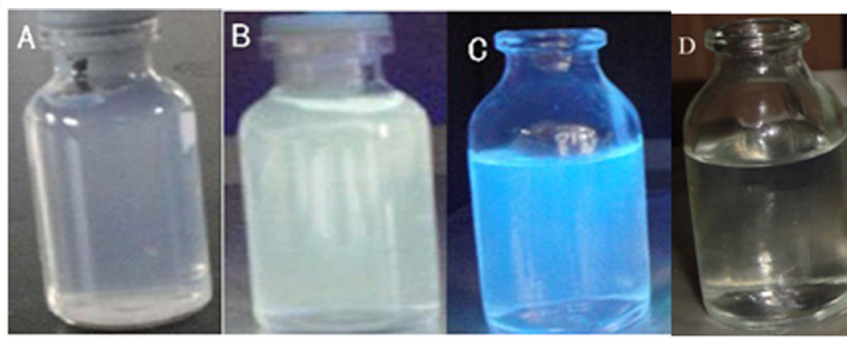

Fig. 2. Digital photographs of $\mathrm{ZnO}$ QDs in chloroform under daylight (A) and under UV lamp $(\lambda=365 \mathrm{~nm})(\mathrm{B})$, and the typical digital photographs of aqueous solution of $\mathrm{ZnO}$ nanoclusters modified by $3.5 \mathrm{G}$ PAMAMs under UV lamp $(\lambda=365 \mathrm{~nm})(\mathrm{C})$ and under daylight (D)

Fig. 3 shows the FTIR spectra of $\mathrm{ZnO}$ QDs and $\mathrm{ZnO}$ nanoclusters modified by $3.5 \mathrm{G}$ PAMAMs. The typical absorption bands of strong ester $\mathrm{C}=\mathrm{O}$ bond at $1738 \mathrm{~cm}^{-1}, \mathrm{C}-\mathrm{N}$ bond at 1090,1055 and $1028 \mathrm{~cm}^{-1}, \mathrm{~N}-\mathrm{H}$ bond at $2962 \mathrm{~cm}^{-1}$, C-H stretch vibration at $2846 \mathrm{~cm}^{-1}, \mathrm{Zn}-\mathrm{N}$ bond at $990 \mathrm{~cm}^{-1}$ and $\mathrm{Zn}-\mathrm{O}$ bond at $586 \mathrm{~cm}^{-1}$ were detected ${ }^{10,25}$. The above characteristic bands indicated that the surface of $\mathrm{ZnO}$ QDs was successfully modified by the PAMAMs.

The typical TEM images of $\mathrm{ZnO}$ nanoclusters modified by 3.5G PAMAMs and ligand-free $\mathrm{ZnO}$ QDs were displayed in Fig. 4. The $\mathrm{ZnO}$ nanoclusters were composed of numerous $\mathrm{ZnO}$ QDs and the size of the corresponding ZnO QD measured from the TEM image (B) was approximately $10 \mathrm{~nm}$ for all samples. Nevertheless, $\mathrm{ZnO}$ nanoclusters presented different sizes, and the size of $\mathrm{ZnO}$ cluster varied from $10 \mathrm{~nm}$ to $60 \mathrm{~nm}$. The HRTEM image of ZnO QDs indicated that the ZnO QDs showed excellent crystallinity, however, $\mathrm{ZnO}$ nanoclusters demonstrated a typical amorphous structure.

Fig. 5 presented the UV-visible absorption spectra of $\mathrm{ZnO}$ nanoclusters in water and $\mathrm{ZnO}$ QDs in $\mathrm{CHCl}_{3}$. The $\mathrm{ZnO}$ nanoclusters exhibited wider and red-shifted optical absorption 


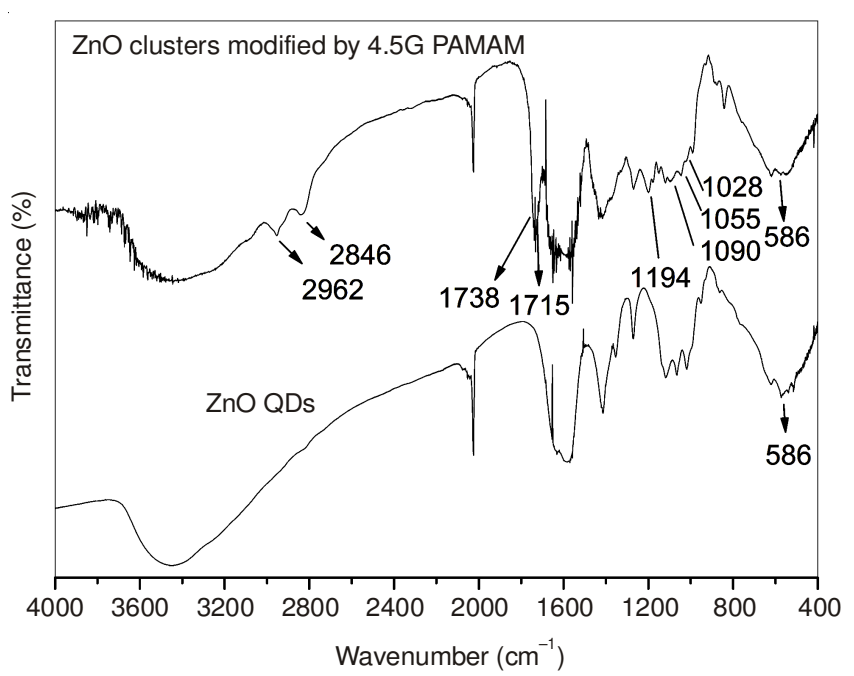

Fig. 3. FTIR spectra of $\mathrm{ZnO}$ nanoclusters modified by $3.5 \mathrm{G}$ PAMAMs and $\mathrm{ZnO}$ QDs
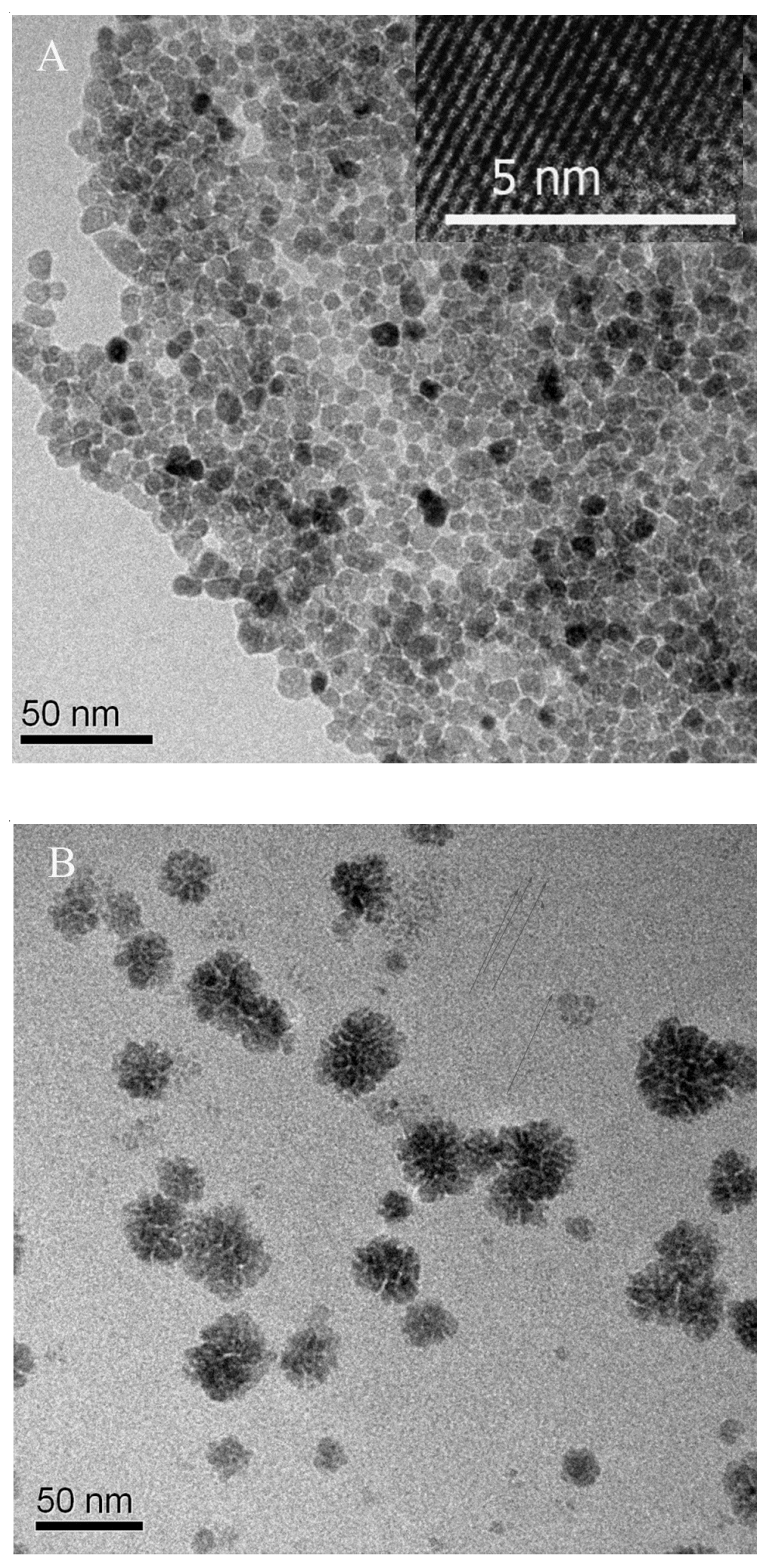

Fig. 4. TEM images of ligand-free ZnO QDs (A, HRTEM (inset)) and ZnO nanoclusters modified by 3.5G PAMAMs (B) spectra comparing with $\mathrm{ZnO}$ QDs (from 284 to $292 \mathrm{~nm}$ ). All absorption curves of $\mathrm{ZnO}$ nanoclusters displayed intensive absorptions in the range 270 to $410 \mathrm{~nm}$, however, $\mathrm{ZnO}$ QDs showed sharper absorptions in the range 270 to $320 \mathrm{~nm}$. This may be attributed to the quantum-size effect of nanoparticles ${ }^{26}$.

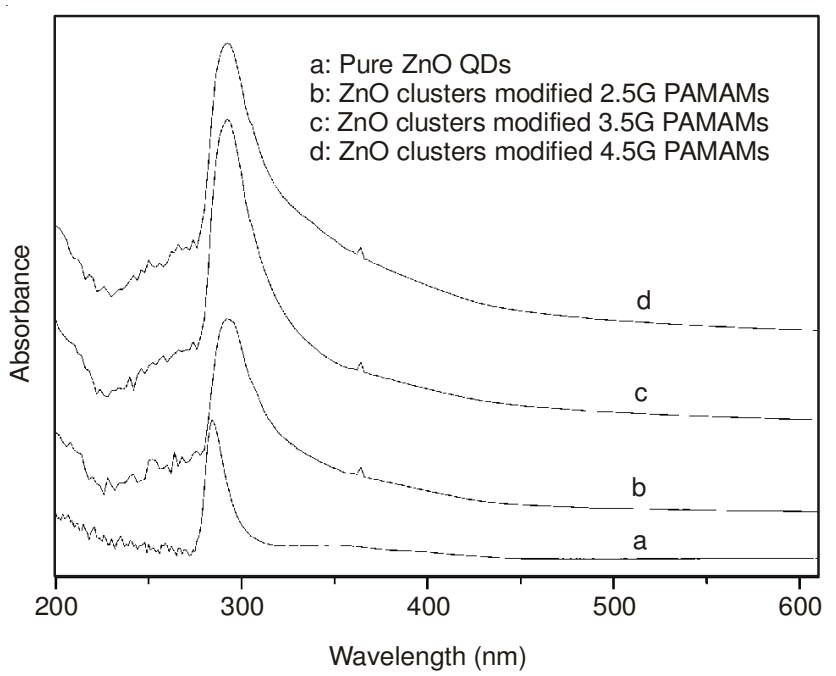

Fig. 5. UV-visible absorption spectra of $\mathrm{ZnO}$ nanoclusters modified by 2.5 G PAMAMs, 3.5G PAMAMs, 4.5G PAMAMs (in water) and $\mathrm{ZnO}$ QDs (in $\mathrm{CHCl}_{3}$ )

Fig. 6 displayed the fluorescent spectra of $\mathrm{ZnO}$ QDs and $\mathrm{ZnO}$ nanoclusters. It was obvious that the emission peaks of the $\mathrm{ZnO}$ nanoclusters were quite different from those of $\mathrm{ZnO}$ QDs. The emission peak at $404 \mathrm{~nm}$ of the $\mathrm{ZnO}$ nanoclusters became weaker comparing with $\mathrm{ZnO}$ QDs, and a new emission peak appeared at $330 \mathrm{~nm}$, which may be the characteristic emission peak of the $\mathrm{ZnO}$ nanoclusters ${ }^{27,28}$.

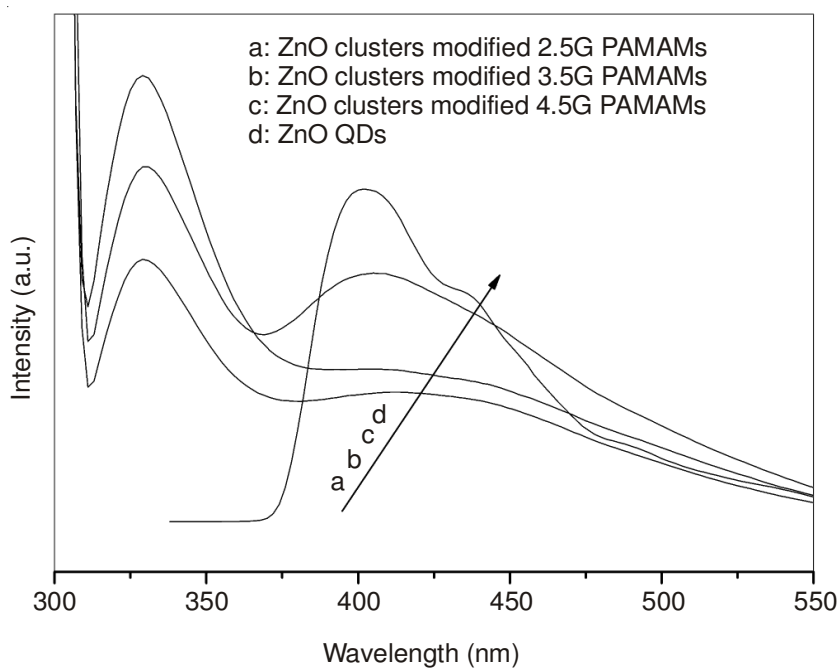

Fig. 6. Fluorescent spectra of $\mathrm{ZnO}$ nanoclusters modified by $2.5 \mathrm{G}$ PAMAMs, 3.5G PAMAMs, 4.5G PAMAM s(in water) and $\mathrm{ZnO}$ QDs (in $\left.\mathrm{CHCl}_{3}\right)(\lambda \mathrm{ex}=300 \mathrm{~nm})$

In order to investigate the application of $\mathrm{ZnO}$ nanoclusters in plastics, we prepared three films of poly(vinyl alcohol) (PVA), PVA/ZnO QDs and PVA/ZnO nanoclusters and all the contents of $\mathrm{ZnO}$ are $2 \mathrm{wt}$. \% in the composite films. Fig. 7 (A) showed the digital photographs of three films of PVA (a), PVA/ 
$\mathrm{ZnO}$ QDs (b) and PVA/ZnO nanoclusters (c) under UV lamp $(\lambda=365 \mathrm{~nm})$. It is obvious that the poly(vinyl alcohol) film did not emit fluorescence, PVA/ZnO QDs film emitted weak yellow fluorescence and $\mathrm{PVA} / \mathrm{ZnO}$ nanoclusters film emitted blue fluorescence. Fig. 7 (B) presented the UV-visible transmittance spectra of pure PVA film and the composite films of $\mathrm{PVA} / \mathrm{ZnO}$ QDs and PVA/ZnO clusters. It is evident that the PVA/ZnO nanoclusters film absorbs nearly $99 \%$ of UV light at wavelengths between 200 and $240 \mathrm{~nm}$ while $75 \%$ between 240 and $300 \mathrm{~nm}$. However, the PVA/ZnO QDs film, absorbs only $80 \%$ of UV light at wavelengths between 200 and 240 nm while $70 \%$ between 240 and $300 \mathrm{~nm}$.
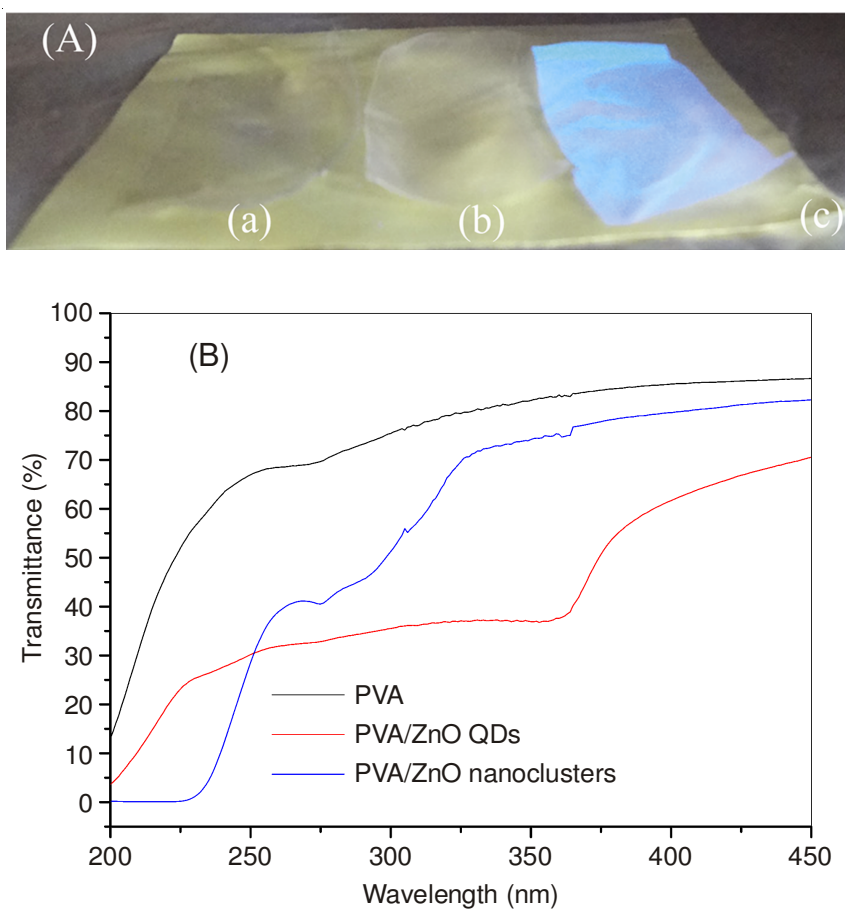

Fig. 7. (A) Digital photographs of films of PVA (a), PVA/ZnO QDs (b) and PVA/ZnO nanoclusters (c) (the background colour: yellow). (B) UV-Visible transmittance spectra of pure poly(vinyl alcohol) film and the composite films of PVA/ZnO QDs and PVA/ZnO clusters)

\section{Conclusion}

Zinc oxide nanoclusters prepared by dissolving of $\mathrm{ZnO}$ QDs in the water solutions of esterifiable PAMAMs are stable under sunlight and show bright blue under UV lamp. TEM measurements showed that the typical $\mathrm{ZnO}$ nanoclusters were made up of $\mathrm{ZnO}$ QDs. FTIR spectra of $\mathrm{ZnO}$ nanoclusters showed the successful modification of ZnO QDs by the esterifiable PAMAMs. The UV-visible absorption spectra indicated that the $\mathrm{ZnO}$ nanoclusters exhibited wider and red-shift optical absorption spectra comparing with ZnO QDs. The fluorescent spectra showed that the emission peaks at $404 \mathrm{~nm}$ of the $\mathrm{ZnO}$ nanoclusters became weaker and the new emission peaks were found at $330 \mathrm{~nm}$ comparing with $\mathrm{ZnO}$ QDs. The UV-visible transmittance spectra showed that the PVA/ZnO nanoclusters film absorbed nearly $99 \%$ of UV light at wavelengths between 200 and $240 \mathrm{~nm}, 75 \%$ between 240 and $300 \mathrm{~nm}$. While the
PVA/ZnO QDs film, absorbs only $80 \%$ of UV light at wavelengths between 200 and $240 \mathrm{~nm}$ while $70 \%$ between 240 and $300 \mathrm{~nm}$.

\section{ACKNOWLEDGEMENTS}

This study was supported by Condition-Renovation Specific Item of Hunan Province (2010TT2052), Key Science and Technology Financing Projects of Ministry of Education (No. 211124) of China, Natural Science Foundation of Hunan Province (No. 11JJ3053), Scientific Research Fund of Hunan Provincial Education Department (No. 10B087) and a grant from Jishou University (jsdxkyzz200904), China.

\section{REFERENCES}

1. S.J. Pearton, D.P. Norton, K. Ip, Y.W. Heo and T. Steiner, Progr. Mater. Sci., 50, 293 (2005).

2. A.M. El-Toni, S. Yin and T. Sato, J. Mater. Sci., 43, 2411 (2008).

3. J.X. Wang, J. Liang, H.M. Wu, W.F. Yuan, Y.Q. Wen, Y.L. Song and L. Jiang, Polym. Int., 57, 509 (2008).

4. Y.-S. Luo, J.-P. Yang, X.-J. Dai, Y. Yang and S.-Y. Fu, J. Phys. Chem. $C, 113,9406$ (2009).

5. C.L. Lü, J.F. Gao, Y.Q. Fu, Y.Y. Du, Y.L. Shi and Z.M. Su, Adv. Funct. Mater., 18, 3070 (2008).

6. S.X. Zhou and L.M. Wu, Macromol. Chem. Phys., 209, 1170 (2008).

7. Y.R. Cheng, C.L. Lü, Z. Lin, Y.F. Liu, C. Guan, H. Lü and B. Yang, J. Mater. Chem., 18, 4062 (2008).

8. Y.L. Wu, A.I.Y. Tok, F.Y.C. Boey, X.T. Zeng and X.H. Zhang, Appl. Surf. Sci., 253, 5473 (2007).

9. Y. Zhang, A.M. Schnoes and A.R. Clapp, ACS Appl. Mater. Interfaces, 2, 3384 (2010).

10. Q. Yuan, S. Hein and R.D.K. Misra, Acta Biomater., 6, 2732 (2010).

11. N. Hagura, T. Ogi, T. Shirahama, F. Iskandar and K. Okuyama, J. Lumin., 131, 921 (2011).

12. D.P. Liu, G.D. Li, Y. Su and J.S. Chen, Angew. Chem. Int. Ed., 45, 7370 (2006).

13. H.M. Xiong, Z.D. Wang and Y.Y. Xia, Adv. Mater., 18, 748 (2006).

14. A. Joseph, G.L. Praveen, K. Abha, G.M. Lekha and S. George, J. Lumin., 132, 1999 (2012).

15. C. Gao and D. Yan, Progr. Polym. Sci., 29, 183 (2004).

16. T. Kim, H.J. Seo, J.S. Choi, H.-S. Jang, J. Baek, K. Kim and J.-S. Park, Biomacromolecules, 5, 2487 (2004).

17. H. Liu, T. Tørring, M. Dong, C.B. Rosen, F. Besenbacher and K.V. Gothelf, J. Am. Chem. Soc., 132, 18054 (2010).

18. C.A. Fail, S.A. Evenson, L.J. Ward, W.C.E. Schofield and J.P.S. Badyal, Langmuir, 18, 264 (2002).

19. Y. Tang, Y.-B. Li, B. Wang, R.-Y. Lin, M. van Dongen, D.M. Zurcher, X.-Y. Gu, M.M. Banaszak Holl, G. Liu and R. Qi, Mol. Pharm., 9, 1812 (2012).

20. V. Maingi, M.V.S. Kumar and P.K. Maiti, J. Phys. Chem. B, 116, 4370 (2012).

21. Y. Higuchi, C. Wu, K.-L. Chang, K. Irie, S. Kawakami, F. Yamashita and M. Hashida, Biomaterials, 32, 6676 (2011).

22. D.A. Tomalia, H. Baker, J.R. Dewald, M. Hall, G. Kallos, S. Martin, J. Roeck, J. Ryder and P. Smith, Polym. J., 17, 117 (1985).

23. D.A. Tomalia, A.M. Naylor and W.A. Goddard, Angew. Chem. Int. Ed., 29, 138 (1990)

24. Y. Tu, L. Zhou, Y.Z. Jin, C. Gao, Z.Z. Ye, Y.F. Yang and Q.L. Wang, J. Mater. Chem., 20, 1594 (2010).

25. M. Sato, A. Kawata, S. Morito, Y. Sato and I. Yamaguchi, Eur. Polym. J., 44, 3430 (2008).

26. Z. Zhang, C.-C. Wang, R. Zakaria and J.Y. Ying, J. Phys. Chem. B, 102, 10871 (1998).

27. H. Zeng, G. Duan, Y. Li, S. Yang, X. Xu and W. Cai, Adv. Funct. Mater., 20, 561 (2010).

28. H. Zeng, W. Cai, P. Liu, X. Xu, H. Zhou, C. Klingshirn and H. Kalt, ACS Nano, 2, 1661 (2008). 